\title{
Cistouretropexia laparoscópica tipo Burch para el tratamiento de la incontinencia urinaria de esfuerzos: seguimiento a largo plazo
}

\author{
Gustavo Calle G. ${ }^{1}$, José De Los Ríos P. ${ }^{1}$, Juan Castañeda R. ${ }^{1}$, Eduardo Serna A. ${ }^{1}$, \\ Ricardo Vásquez R. ${ }^{1}$, Adriana Arango M. ${ }^{2}$, Henry Muñoz S. ${ }^{2}$, Carlos Buitrago D. ${ }^{2}$ \\ 1 Unidad de Endoscopia Ginecológica, Clínica del Prado, Universidad CES. \\ 2 Programa de Endoscopia Ginecológica, Universidad CES. Medellín, Colombia.
}

\section{RESUMEN}

Objetivos: Conocer las complicaciones, las tasas de cura subjetiva y valoración con el índice de severidad de Sandvik a largo plazo de pacientes en quienes se realizó cistouretropexia laparoscópica tipo Burch para la corrección de su incontinencia urinaria de esfuerzo (IUE). Método: Análisis descriptivo, retrospectivo. Nivel de evidencia III. Se incluyeron las pacientes con diagnóstico de IUE tipo Blaivas IIB, a quienes se les practicó Burch laparoscópico en la Clínica del Prado y Profamilia Medellín, entre los años 2003 y 2007. Las variables principales a evaluar fueron edad, tiempo quirúrgico, cura subjetiva, índice de severidad de Sandvik y complicaciones. Resultados: Se analizaron 72 pacientes con promedio de edad de 46,57 $\pm 9,54$ años. El tiempo promedio de seguimiento fue de 24,4 meses (rango: 9,0-55,8 meses). El tiempo quirúrgico promedio fue de 79,83 minutos (rango: 44-160 minutos). Refirieron cura subjetiva 56 pacientes $(77,8 \%)$. El índice de severidad de Sandvik muestra que a largo plazo $56,9 \%$ pacientes estaban secas con remisión completa de los síntomas y el $13,8 \%$ pacientes tenían pérdidas leves. Conclusiones: El Burch laparoscópico es una opción efectiva para el manejo de la incontinencia urinaria de esfuerzo tipo Blaivas IIB. Provee tasas de curación subjetiva a largo plazo similares a otros tipos de tratamiento. Es una opción más a tener en cuenta en el manejo de la IUE, en especial aquellas pacientes con patologías asociadas que se beneficien del abordaje por laparoscopia.

\section{PALABRAS CLAVE: Incontinencia urinaria de esfuerzo, colposuspensión laparoscópica de Burch, índice de Sandvik}

\section{SUMMARY}

Objectives: To describe the subjective cure rate, the score of the Sandvik test and the complications after laparoscopic Burch in patients with stress urinary incontinence (SUI). Methods: Prospective descriptive study. We included all patients diagnosed with SUI Blaivas type IIB, and operated on by laparoscopy in two reference institutions between 2003 and 2007. Results: We analyzed 72 patients with an average age of 46.57 \pm 9.54 years. The average follow-up was 24.4 months, with a minimum of 9 and a maximum of 55.8. The average operating time in 29 patients in which we only performed laparoscopic Burch with or without colporrhaphy was 93.7 minutes (55-180 minutes); 56 patients (77.8\%) reported subjective cure. The Sandvik`s severity index found $56.9 \%$ dry patients and $13.8 \%$ with mild losses. Conclusion: The laparoscopic management may be useful in some patients, especially those who will be carried to laparoscopy by some other indication. Subjective cure rates are similar to other types of surgery.

KEY WORDS: Stress urinary incontinence, laparoscopic Burch colposuspension, Sandvik`s index 


\section{INTRODUCCIÓN}

La incontinencia urinaria de esfuerzo (IUE) afecta entre el 15 y $28 \%$ de las mujeres. Factores como la edad, el número de embarazos y partos, la actividad laboral, y las enfermedades de base predisponen a las mujeres a presentar esta patología gineco-urológica (1).

Vancaillie y Schuessler, en 1991, describieron la colposuspensión laparoscópica tipo Burch en un intento por minimizar el tamaño de las incisiones, disminuir la estancia hospitalaria y agilizar el retorno a las actividades normales, con respecto a la cirugía convencional por laparotomía (2). Ulmstein y cols (3), cinco años después, describen la técnica para la aplicación de la cintilla vaginal de uretra media sin tensión (TVT) de fácil aplicación y al parecer tan eficaz como la colposuspensión.

El Grupo Cochrane en su revisión del 2006, comparó el Burch laparoscópico con el TVT para el tratamiento de la IUE (4). En este metanálisis, las pacientes del grupo de TVT presentaron menos fugas en pruebas objetivas (RR: 0,92; IC 95\%: $0,85-0,99)$, pero no hubo diferencia significativa en la tasa de curación subjetiva al comparar los dos grupos. Una limitación importante para medir el desenlace fue el corto seguimiento posoperatorio en ambos grupos, de tan sólo 18 meses, por lo que recomiendan la realización de más estudios.

A pesar de la actual tendencia a usar las técnicas con malla por el menor tiempo quirúrgico empleado, hay que recordar que éstas no están exentas de complicaciones. Se han reportado dificultades para el vaciamiento vesical, lesiones uretrales, vesicales y vasculares, extrusión de la malla, molestias locales o aparición de incontinencia de urgencia de novo, entre otras. La disfunción miccional es la complicación más frecuente, encontrándose hasta en el 16,3\% de las pacientes operadas por esta técnica (5).

En Colombia, el costo de la malla no está al alcance de la mayoría de la población y tampoco es asumido por las empresas aseguradoras de salud, por lo cual en nuestro medio, se mantienen vigentes procedimientos como la cistouretropexia. Las tasas de éxito reportadas con la técnica laparoscópica son similares a las de las técnicas abiertas (4), por lo que no existe una razón de peso para excluirla del arsenal terapéutico con el que la medicina actual enfrenta la IUE.

Este trabajo compila la descripción de las variables del procedimiento y los resultados a largo plazo de una serie de casos de cistouretropexia laparoscópica tipo Burch.

\section{PACIENTES Y MÉTODOS}

Se realizó un estudio descriptivo y retrospectivo, revisando 105 historias clínicas de pacientes con IUE tipo Blaivas IIB, a quienes se les realizó cistouretropexia laparoscópica tipo Burch, entre febrero de 2003 y diciembre de 2007, en 2 centros de referencia para cirugía endoscópica ginecológica de la ciudad de Medellín, Colombia (Clínica del Prado y Profamilia). Se incluyeron 72 pacientes para el análisis. Las demás fueron excluidas por las siguientes causas: 29 no lograron ser contactadas telefónicamente, 2 tenían información incompleta en la historia clínica y 2 no desearon participar en el estudio. De la historia clínica se obtuvieron las variables del acto quirúrgico y complicaciones perioperatorias; el resto de las variables se evaluó mediante un cuestionario diligenciado telefónicamente en las pacientes que consintieron participar en la investigación. La tasa de curación subjetiva fue determinada por la respuesta dada por la paciente a la siguiente pregunta: ¿Considera que la cirugía le resolvió su problema de incontinencia? El índice de severidad de Sandvik $(6,7)$ valora la severidad de la IUE después de asignar un puntaje a las siguientes dos preguntas: ¿Que tan a menudo ha experimentado pérdida de orina después de la cirugía? $(0=$ ninguna, 1= menos de una vez al mes, 2=1 o más veces al mes, $3=1$ o más veces a la semana, $4=$ todos los días o todas las noches). ¿Qué cantidad de orina pierde? $(0=$ Nada, $1=$ Gotas 0 poco, $2=$ Más). El puntaje de la primera pregunta se multiplica por la segunda resultando en: $0=$ seca, $1-2=$ incontinencia leve, 3-4= incontinencia moderada, 6-8 = incontinencia severa.

Variables a medir: 1. Datos generales de las pacientes: edad, peso. 2. Características del procedimiento: tiempo quirúrgico (contabilizado desde la inserción de la aguja de Veress hasta la extracción del último trócar o en caso de colporrafia, hasta la finalización de ésta). 3. Cura subjetiva, referida por la paciente. 4. Índice de severidad de Sandvik. 5. Complicaciones intraoperatorias (lesión vesical, vascular, lesión de intestino, sangrado excesivo, necesidad de laparotomía) y postoperatorias (infección de vías urinarias, incontinencia urinaria de urgencia de novo, hematoma o infección de herida o sitio quirúrgico).

Con la información obtenida se construyó una base de datos. Las variables continuas se describen por medio de medidas de tendencia central y dispersión, las categóricas por medio de proporciones.

Descripción de la técnica quirúrgica utilizada: 
El Burch laparoscópico fué realizado con la técnica intraperitoneal, con un puerto umbilical de $10 \mathrm{~mm}$ para la colocación de la cámara y cuatro puertos adicionales, tres de $5 \mathrm{~mm}$ en fosas iliacas y flanco izquierdo, y otro de $10 \mathrm{~mm}$ supra púbico. Se realiza inicialmente la apertura de los espacios de Retzius desde la línea media, $3 \mathrm{cms}$ por encima del borde superior de la vejiga. Se diseca lateralmente en forma bilateral, con la asistencia del bipolar, hasta identificar el ligamento de Cooper y el arco tendinoso de la pelvis, de aspecto nacarado; con la elevación de vagina retropúbica y parauretral digitalmente, por el mismo cirujano, se coloca un punto de material no absorbible trenzado y se fija al ligamento de Cooper bilateral en forma similar a la técnica abierta. Se eleva el paracolpos y se tensionan los puntos levemente por encima del borde superior de la rama descendente del pubis, colocando 1 punto a cada lado. Se revisa la hemostasia y el peritoneo parietal no es afrontado.

Las pacientes a quienes se les realizó el Burch como único procedimiento fueron dadas de alta en el mismo día operatorio, sin sonda vesical y con analgésicos orales.

\section{RESULTADOS}

Se analizó la información en 72 pacientes que cumplieron los criterios de inclusión. La edad promedio fue de 46,5 \pm 9,54 años con (rango: 24-73 años). El tiempo promedio de seguimiento fue de 24,4 meses (rango: 9,0-55,8 meses). El promedio del tiempo quirúrgico utilizado en el total de pacientes fue de 120 minutos (rango: 55-265 minutos). En este cálculo se incluyeron todos los procedimientos concomitantes realizados, de los cuales el más común fue histerectomía laparoscópica total. Sin embargo, cuando se analizó el tiempo quirúrgico de las pacientes a quienes se les practicó Burch solamente o Burch con plastía posterior (10 y 19 pacientes respectivamente), el tiempo operatorio promedio fue de 79,83 minutos (rango: $44-160 \mathrm{mi}-$ nutos).

El $77,8 \%$ de las pacientes refirieron cura subjetiva (n: 56). De acuerdo al índice de severidad de Sandvik, 56,9\% corresponden al grupo de secas y $13,8 \%$ pérdidas leves, para un total de $70,7 \%$ sin pérdidas importantes (Tabla I).

Se encontró que a 4 de las pacientes que habían respondido sentirse curadas de manera subjetiva $(28,6 \%)$, cuando se les aplicó el índice de Sandvik, este las catalogó como teniendo incontinencia urinaria severa. La Tabla II muestra la correlación obtenida en el estudio entre las tasas de curación subjetiva y el índice de severidad de Sandvik.
Tabla I

EVALUACIÓN DEL ÍNDICE DE SEVERIDAD DE SANDVIK

\begin{tabular}{lrr}
\hline Índice de severidad & $\mathrm{n}$ & \multicolumn{1}{c}{$\%$} \\
\hline Seca & 41 & 56,9 \\
Leve & 10 & 13,9 \\
Moderada & 7 & 9,7 \\
Severa & 14 & 19,4 \\
Total & 72 & 100 \\
\hline
\end{tabular}

Tabla II

RELACIÓN DE RESULTADOS ENTRE ÍNDICE DE SEVERIDAD DE SANDVIK Y CURA SUBJETIVA

\begin{tabular}{lccccc}
\hline & \multicolumn{5}{c}{ Cura subjetiva } \\
Índice de Sandvik & Si & $\%$ & No & $\%$ & Total \\
\hline Seca & 41 & 100 & 0 & 0 & 41 \\
Leve & 10 & 100 & 0 & 0 & 10 \\
Moderada & 1 & 14,3 & 6 & 85,7 & 7 \\
Severa & 4 & 28,6 & 10 & 71,4 & 14 \\
Total & 56 & 77,8 & 16 & 22,2 & 72 \\
\hline
\end{tabular}

Se presentaron complicaciones posoperatorias en 5 pacientes $(6,94 \%), 3$ hematomas pélvicos en pacientes con histerectomía concomitante y 2 infecciones del tracto urinario. No se presentaron complicaciones durante la realización de la cirugía. Hasta la fecha de recolección de la información, 6 pacientes $(8,3 \%)$ habían sido llevadas a una nueva cirugía para incontinencia urinaria.

\section{DISCUSIÓN}

Hace más de dos décadas, se presentaron los primeros resultados del Burch por laparoscopia, demostrando que era un tratamiento eficaz para la incontinencia urinaria de esfuerzos (5). El metanálisis del grupo Cochrane encontró que este procedimiento es equivalente a la cirugía de Burch por laparotomía con respecto a las tasas de cura subjetiva (4). Otros ensayos aleatorios posteriores no han identificado diferencia significativa entre los dos procedimientos con respecto a las tasas de curación subjetiva u objetiva $(8,9)$. Hay más de 150 reportes de estudios utilizando la vía laparoscópica. 
Tabla III COMPARACIÓN DE LOS RESULTADOS DE BURCH LAPAROSCÓPICO EN LA LITERATURA*,
SEGÚN EL ÍNDICE DE SEVERIDAD DE SANDVICK

\begin{tabular}{|c|c|c|c|c|c|c|}
\hline Estudio & $\mathrm{n}$ & $\begin{array}{c}\text { Seguimiento } \\
\text { meses }\end{array}$ & $\begin{array}{c}\text { Cura } \\
\text { subjetiva } \\
\%\end{array}$ & $\begin{array}{c}\text { Cura } \\
\text { objetiva } \\
\%\end{array}$ & $\begin{array}{c}\text { Complicaciones } \\
\%\end{array}$ & $\begin{array}{l}\text { Tiempo } \\
\text { quirúrgico } \\
\text { minutos }\end{array}$ \\
\hline Dean et al. (4) & & 18 & $62-100$ & & & \\
\hline Kitchener et al. (8) & 240 & 24 & 55 & 80 & 14,1 & 65 \\
\hline Carey et al. (9) & 200 & 24 & 63 & NA & NA & 87 \\
\hline Ankardal et al. (12) & 75 & 12 & 88 & 90 & 12 & 84 \\
\hline Yang et al. (13) & 116 & 12 & 96 & 95 & 1,9 & NA \\
\hline Bunyavejchevin et al. (14) & 21 & 60 & NA & 76 & 19 & 70 \\
\hline Dietz et al. (15) & 50 & 12 & 74 & NA & NA & NA \\
\hline Clínica Prado 2009 & 72 & 24 & 77,8 & 70,7 & 6,9 & 79,8 \\
\hline
\end{tabular}

* Modificación de Tabla publicada por Jenkins TR \& Liu CY (10).

Estudios de cohortes prospectivos han encontrado tasas de curación que van del 76 al 95\% $(10,11)$.

Los diferentes estudios han mostrado que el Burch por laparoscopia requiere un mayor tiempo quirúrgico que la cirugía a cielo abierto, pero la menor pérdida sanguínea, la disminución de dolor posoperatorio, y un más rápido retorno a las actividades normales que son ventajas propias de los procedimientos laparoscópicos, también han sido demostradas en el Burch por laparoscopia (10). Al comparar las tasas de curación, tiempo quirúrgico y complicaciones con otras series, encontramos resultados similares (Tabla III).

Las diferencias en las tasas reportadas de curación objetiva se deben en su mayoría a las diversas formas que los autores utilizan para definir esta variable. Consideramos que en una enfermedad como la IUE, donde por definición la paciente es la principal testigo y evidencia de su propio padecimiento y mejoría, debe privilegiarse la tasa de curación subjetiva referida por la paciente sobre cualquier dato urodinámico obtenido.

\section{CONCLUSIÓN}

El Burch laparoscópico es una opción efectiva para el manejo de la IUE tipo Blaivas IIB. Provee tasas de curación subjetiva a largo plazo similares a otros tipos de tratamiento. Es una opción más a tener en cuenta en el manejo de la IUE, en especial aquellas pacientes con patologías asociadas que se beneficien del abordaje por laparocopia.

\section{BIBLIOGRAFÍA}

1. Aristizábal Agudelo JM. Deficiencia esfinteriana intrínseca. En: Lomanto Morán A. Incontinencia urinaria femenina. Bogotá D.C: Editorial Litocamargo Ltda. Marzo 2001. p: 337-47.

2. Vancaille T, Schuessler W. Laparoscopic bladder neck suspension. J Laparoendosc Surg 1991;1(3):169-73.

3. Ulmsten U, Henriksson L, Johnson P, Varhos G. An ambulatory surgical procedure under local anesthesia for treatment of female urinary incontinence. Int Urogynecol J Pelvic Floor Dysfunct 1996;7:81-5.

4. Dean NM, Ellis G, Wilson PD, Herbison GP. Laparoscopic colposuspension for urinary incontinence in women. Cochrane Database Syst Rev 2006;3:CD002239. Date of most recent substantive amendment: 31 October 2007

5. Stanford EJ, Paraiso MF. A comprehensive review of suburethral sling procedure complications. J Minim Invasive Gynecol 2008;15(2):132-45.

6. Sandvik H, Hunskaar S, Seim A, Hermstad R, Vanvik $A$, Bratt $H$. Validation of a severity index in female urinary incontinence and its implementation in an epidemiological survey. J Epidemiol Community Health 1993;47:4979.

7. Hanley J, Capewell A, Hagen S. Validity study of the severity index, a simple measure of urinary incontinence in women. BMJ 2001;322:1096-7.

8. Kitchener HC, Dunn G, Lawton V, Reid F, Nelson L, Smith AR; COLPO Study Group. Laparoscopic versus open colposuspension--results of a prospective randomized controlled trial. BJOG 2006;113(9):1007-13.

9. Carey M, Goh JT, Rosamilia A, Cornish A, Gordon I, Hawthorne G, Maher CF, Dwyer PL, Moran P, Gilmour DT. Laparoscopic versus open Burch colposuspension: a randomized controlled trial. BJOG 2006;113(9):999-1006. 
10. Jenkins TR, Liu CY. Laparoscopic Burch colposuspension. Curr Opin Obstet Gynecol 2007;19(4):314-8.

11. Hong JH, Choo MS, Lee KS. Long-Term Results of Laparoscopic Burch Colposuspension for stress urinary incontinence in women. J Korean Med Sci 2009; 24(6):1182-6.

12. Ankardal M, Milson I, Stjerndahl JH, Engh ME. A three-armed randomized trial comparing open Burch colposuspension using sutures with laparoscopic colposuspension using mesh and staples in women with stress urinary incontinence. Acta Obstet Gynecol Scand 2005;84:773-9.
13. Yang JM, Yang SH, Huang WC. A surgical technique to adjust bladder neck suspension in laparoscopic Burch colposuspension. J Minim Invasive Gynecol 2006;13:289-95.

14. Bunyavejchevin S, Wisawasukmongchol W. Five years follow up of laparoscopic Burch colposuspension for stress urinary incontinence in Thai women. $J$ Med Assoc Thai 2005;88:1182-86.

15. Dietz HP, Wilson PD. Laparoscopic colposuspension versus urethropexy: a case-control series. Int Urogynecol J 2005;16:15-8. 